\title{
Influencia de un programa de actividad física sobre la atención selectiva y la eficacia atencional en escolares \\ Influence of a physical activity program on selective attention and attentional efficiency in school children \\ Andres Rosa Guillamón, Eliseo Garcia Canto, Hector Martínez García \\ Universidad de Murcia (España)
}

\begin{abstract}
Resumen. La atención se ha revelado como un mecanismo de control que desempeña un rol fundamental en la organización jerárquica de procesos no solo neurocognitivos sino también motivacionales y emocionales. La reciente evidencia científica que apoya la existencia de una relación directa entre ejercicio físico y atención demanda la necesidad de definir las premisas para el diseño de intervenciones con el objetivo de examinar qué tipo de actividad física puede influir de manera más efectiva en la evolución cognitiva preadolescente subyacente al ejercicio físico. El objetivo fue analizar el efecto sobre la atención de un programa de actividad física de alta intensidad. Se diseñó un estudio cuasi-experimental intrasujetos en el que participaron 24 niños y 20 niñas de 9-10 años pertenecientes a España. Se evaluó la atención mediante el Test de Caras. Se aplicó estadística no paramétrica para el análisis de datos (prueba de Wilcoxon). Se apreciaron efectos sobre las variables de aciertos, errores, omisiones, control inhibitorio y eficacia atencional (p entre $.05 \mathrm{y}<.001$ ). Las diferencias se mantuvieron cuando se realizó un análisis diferenciado por sexo, excepto para las niñas en errores $(\mathrm{p}>.05)$. Los resultados sugieren que la realización de un programa de actividad física de alta intensidad, con 16 sesiones de 40-50 minutos de duración/sesión, basado en ejercicios de fuerza-resistencia muscular y desafíos cooperativos podría tener efectos positivos sobre la atención tanto en niños como en niñas de 9-10 años de edad.
\end{abstract}

Palabras clave: actividad física, educación física, atención selectiva, eficacia atencional, infancia, escolares.

\begin{abstract}
Attention has been revealed as a control mechanism that plays a fundamental role in the hierarchical organization of processes that are not only neurocognitive but also motivational and emotional. Recent scientific evidence supporting the existence of a direct relationship between physical exercise and attention demands the need to define the premises for the design of interventions with the aim of examining which type of physical activity can more effectively influence preadolescent cognitive evolution underlying physical exercise. The objective was to analyze the effect of a high intensity physical activity program on attention. A quasi-experimental intrasubject study was designed. 24 boys and 20 girls aged 9-10 years old from Spain participated in the study. Attention was evaluated through the «Face Test». Non-parametric statistics were applied for the data analysis (Wilcoxon test). Effects were observed on the variables of successes, errors, omissions, inhibitory control, and attentional efficacy ( $\mathrm{p}$ between $<.05$ and $<.001$ ). The differences were maintained when a differentiated analysis was performed by sex, except for girls in errors $(p>.05)$. The results suggest that the realization of a program of physical activity of high intensity, with 16 sessions of 40-50 minutes per session, based on muscular strengthendurance exercises and cooperative challenges, could have positive effects on attention in both boys as girls of 9-10 years of age. Keywords: physical activity, physical education, selective attention, attention efficiency, childhood, schoolchildren.
\end{abstract}

\section{Introducción}

La evidencia científica disponible demuestra el interés entre psicólogos evolutivos, educadores y científicos por conocer los procesos de aprendizaje y desarrollo madurativo en la infancia (Goodway, Ozmun, Gallahue, \& 2020; Korzeniowski, 2011; Piaget \& Inhelder, 2015), ya que se trata de una etapa de la vida muy importante en la construcción de la personalidad, y en la adopción de conductas de estilo de vida que pueden influir de manera determinante en el desarrollo de la persona y en su ajuste psicosocial (Rosa, García, \& Carrillo, 2018a,b).

En esta línea, recientes estudios han puesto el foco de interés en procesos cognitivos tales como la flexibilidad mental, la plasticidad cognitiva, el funcionamiento ejecutivo, el procesamiento del lenguaje, la memoria de trabajo, la concentración, la conciencia plena, la atención (Chen, Zhang, Callaghan, LaChappa, Chen, \& He, 2017; De Bruin, Van del Zwan, \& Bögels, 2016; Haapala, 2012; Khan \& Hillman, 2014; León, 2008; Monteoliva, Ison, \& Pattini, 2014; Pontifex et al., 2011; Scudder et al., 2014), e incluso la calidad del sueño

Fecha recepción: 30-12-19. Fecha de aceptación: 20-03-20

Eliseo Garcia Canto

eliseo.garcia@um.es

\section{(López, López, \& Díaz, 2016).}

Entre todos ellos, la atención se ha revelado como un mecanismo de control que desempeña un rol fundamental en la organización jerárquica de procesos no solo neurocognitivos sino también motivacionales y emocionales (Folgado dos Santos et al., 2020; Ison, Greco, Korzeniowski \& Morelato, 2015; León, 2008). Con las debidas precauciones debido a la complejidad tanto conceptual como anatómica y funcional de la atención, esta se puede definir como una función neurocognitiva que permite gestionar el procesamiento de la información a partir de procesos de percepción, análisis y ejecución; implicando un control voluntario, intencional y consciente de otros procesos psicológicos con el objetivo de llevar a cabo eficazmente una tarea (Monteoliva, Carrada, \& Ison, 2017).

Esta complejidad ha hecho que se diferencien distintos componentes o mecanismos dentro de la atención (Petersen \& Posner, 2012; Sohlberg \& Mateer, 2001). Maureira \& Flores (2016) señalan los mecanismos de estado de alerta, atención selectiva, atención sostenida, atención alternante y atención dividida.

De estos mecanismos, la función selectiva ha sido descrita, en general, como un predictor de éxito en el desarrollo evolutivo infantil (Giuliano, Karns, Neville, \& Hillyard, 2014) $\mathrm{y}$, en particular, en el rendimiento académico, sobre todo, en 
la adquisición de la lectura y la escritura (León, 2008).

Desde otros modelos teóricos se ha formulado un nuevo concepto directamente asociado a la atención selectiva, la eficacia atencional, entendida como la capacidad cognitiva que permite la optimización y regulación en tareas que exigen el empleo conjunto de habilidades de mantenimiento y selectividad atencional (Monteoliva et al., 2017).

En este punto resulta evidente que, durante los últimos años, la atención ha despertado un gran interés en el ámbito educativo, por su implicación en el rendimiento cognitivo, la concentración, la adquisición de conocimiento (Altenburg, Chinapaw, \& Singh, 2016; Chen et al., 2017; Ortiz \& Ramírez, 2018; Rosa, García, \& Carrillo, 2019; Tine, 2014), y en la creciente prevalencia de trastornos de la atención e hiperactividad (López et al., 2015; Villa de Gregorio, Ruiz, \& Barriopedro, 2019).

El desarrollo de la atención es, por tanto, fundamental desde edades tempranas, y se muestra como un proceso gradual y evolutivo que puede verse influenciado por factores ambientales, culturales, educacionales y conductuales (Monteoliva et al., 2017).

En este sentido, existen programas orientados al entrenamiento tanto específico como inespecífico de la atención, así como a la compensación de las dificultades atencionales (Rodríguez, Alexey, Castro, \& Castro, 2008). Algunas de estas intervenciones han incluido la realización de ejercicio físico ya sea de forma aguda o crónica (Maureira \& Flores, 2017), sobre la creencia de que la mejora de las funciones neuropsicológicas asociadas al estado de condición física puede estimular la atención o alguna de sus funciones, a través de procesos como la activación cerebral, el incremento dendritas neuronales, la mejora del riego sanguíneo, la neurogénesis, o el aumento de glías y neurotransmisores cerebrales (Chaddock et al., 2011; Fedewa \& Ahn, 2011; Hillman, 2016; Kashihara, Maruyama, Murota, \& Nakahara, 2009; Maureira, 2016; Pérez et al., 2014).

La reciente evidencia científica que apoya la existencia de una relación directa entre ejercicio físico y atención demanda la necesidad de definir las premisas para el diseño de intervenciones con el objetivo de examinar qué tipo de actividad física puede influir de manera más efectiva en la evolución cognitiva preadolescente subyacente al ejercicio físico (Gallego, Reigal, Hernández, \& Juárez, 2015; Reloba-Martínez et al., 2017), lo que contribuirá al desarrollo de un conocimiento científico coherente (Conde \& Tercedor, 2015).

Estudios precedentes han puesto de relieve la necesidad de diseñar intervenciones bajo las premisas de individualización, especificidad y progresividad en la carga (Rodríguez et al., 2008). No obstante, en un trabajo se observaron efectos tras aplicar tan solo una sesión de 12 minutos de ejercicio físico (Tine, 2014). Otras investigaciones señalan la necesidad de prolongar la intervención para mejorar la capacidad aeróbica, ya que ha sido descrita como la principal responsable en tareas cognitivas que requieren de un alto desempeño atencional (Chaddock et al., 2011; Chen et al., 2017; Pontifex et al., 2011; Wu et al., 2011). Estudios recientes han puesto el foco de atención en ejercicios físicos de carácter explosivo y alta intensidad intermitente frente a otra metodología aeróbica de moderada intensidad, observando resultados positivos sobre la atención selectiva y el span atencional (Reloba-
Martínez et al., 2017).

Sin embargo, algunos estudios ponen en duda los efectos de algunos tipos de ejercicio físico sobre el desempeño cognitivo y atencional. Schmidt et al. (2016), con un programa de tres semanas, y Wilson et al. (2016), con otro de cuatro semanas, no observaron efectos sobre la atención tras aplicar programas basados en pausas activas durante la jornada lectiva. Incluso en un estudio se observó un descenso tanto en la atención selectiva como dividida tras aplicar un programa de cuatro semanas basado en CrossFit ${ }^{\circledR}$ (Perciavalle et al., 2016).

En respuesta al conocimiento científico disponible, en este estudio se diseñó e implementó un programa de actividad física de alta intensidad progresiva, adaptado a las características cognitivo-motrices de los participantes, descritas en el estadio de las operaciones concretas de Piaget \& Inhelder (2015). Se emplearon ejercicios físico-lúdicos que motivan al alumnado, y que son potencialmente efectivos para la mejora de la condición física (Gómez-Mármol, SánchezAlcaraz, Pérez, \& Ribes, 2014; Sánchez-Alcaraz \& GómezMármol, 2015).

También se utilizaron juegos basados en desafíos cooperativos, que fomentan la resolución de problemas motores mediante la implicación cognitiva, la motivación de unos miembros del grupo sobre otros para hallar la solución al problema, el compromiso individual y colectivo, la toma de decisiones, el desarrollo de habilidades de respeto y tolerancia y la igualdad de oportunidades; todo ello genera beneficios a nivel no solo a nivel cognitivo-motor sino también socio-emocional y, en consecuencia, el participante se muestra más activo e implicado (Fernández-Río \& MéndezGiménez, 2016; Slavin, 1992).

Sobre la base de lo anterior, el objetivo de este estudio fue analizar el efecto sobre la atención (especialmente la atención selectiva y la eficacia atencional), de un programa de actividad física de alta intensidad en una muestra de escolares españoles de 9-10. años.

\section{Material y método}

\section{Participantes}

Un total de 98 escolares de 9-10 años de edad (50 niños y 48 niñas) pertenecientes a un colegio público de la Región de Murcia (sur-este de España) fueron, inicialmente, invitados a participar en esta investigación (figura 1). El colegio fue seleccionado por conveniencia. Se solicitó el consentimiento expreso del equipo directivo y la asociación de padres, así como el consentimiento informado de las familias. Los participantes fueron seleccionados de manera no aleatoria y por conveniencia. Estos escolares cursaban tercero o cuarto de educación primaria. Se plantearon los siguientes criterios de inclusión en el estudio: 1) tener una asistencia mínima al colegio del $80 \%$ de las clases lectivas durante el curso escolar; 2) no tener un problema médico u ortopédico que impidiese la participación en el programa de actividad física; 3 ) no presentar antecedentes de patologías neurológicas, trastornos visuales o auditivos, o tratamiento farmacológico; y 4) aportar el consentimiento informado de la familia. Los criterios de exclusión definitivos de aquellos que iniciaron el programa fueron los siguientes: 1) tener un porcentaje de 
asistencia al programa de actividad física superior al $90 \%$ de las sesiones; 2) no cumplimentar el cuestionario de atención pretest y postest.

Tras la aplicación de los criterios de inclusión, de esos 98 escolares inicialmente seleccionados, se escogieron 48 participantes que comenzaron con el programa de intervención. Finalmente, de esos 48 escolares, solamente 24 niños (13 niños de 9 años y 10 niños de 10 años) y 20 niñas (10 niñas de 9 años y 10 niñas de10 años) participaron del estudio, una vez aplicados los criterios de exclusión.

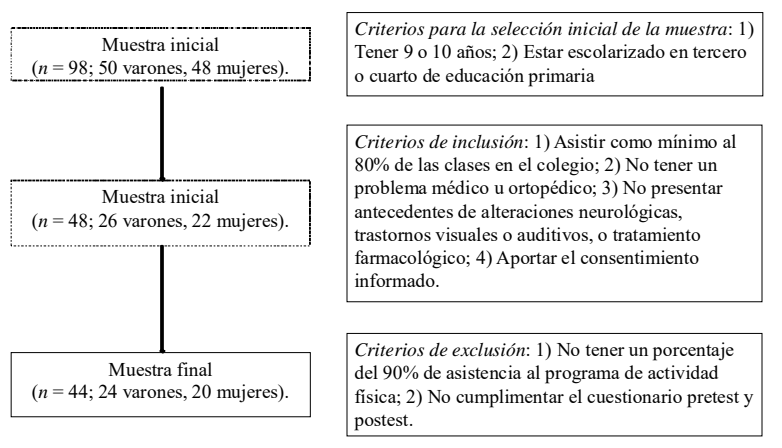

Figura 1. Flujograma de selección de la muestra

\section{Diseño del estudio}

Se empleó un diseño de estudio cuasi-experimental intrasujetos con medidas pre-post (Ato, López, \& Benavente, 2013). No se planteó un grupo de control ya que la intervención se realizó en horario lectivo, evitando entrar en conflicto con las familias de los escolares no participantes del programa. El estudio se desarrolló durante el curso académico 2018/19 (figura 2).

\begin{tabular}{|c|c|}
\hline \begin{tabular}{|l|} 
Fase I: Contacto con el centro \\
29 de octubre de 2018 \\
\end{tabular} & $\begin{array}{l}\text { Confirmación de la participación } \\
12 \text { de noviembre } \\
\end{array}$ \\
\hline $\begin{array}{l}\text { Fase III: Diseño del programa } \\
\text { Del } 27 \text { de noviembre al } 14 \text { de diciembre. }\end{array}$ & $\begin{array}{l}\text { Estudio piloto con escolares no participantes } \\
17,18,19 \text { y } 20 \text { de diciembre. }\end{array}$ \\
\hline $\begin{array}{l}\text { Fase II: Selección de la muestra } \\
19 \text { de noviembre de } 2018 .\end{array}$ & $\begin{array}{l}\text { Confirmación de los participantes } \\
27 \text { de noviembre. }\end{array}$ \\
\hline $\begin{array}{l}\text { Fase IV: Desarrollo de la intervención } \\
\text { Pretest: } 9 \text { de enero. } \\
\text { Programa de actividad física (16 sesiones): } \\
\text { del } 14 \text { de enero al } 8 \text { de marzo. } \\
\text { Postest: } 12 \text { de marzo. } \\
\end{array}$ & $\begin{array}{l}\text { Recogida de datos } \\
\text { Del } 13 \text { al } 24 \text { de marzo. } \\
\text { Comunicación de los resultados al centro } \\
25 \text { de marzo. }\end{array}$ \\
\hline
\end{tabular}

Figura 2. Resumen de las fases del estudio.

\section{Aspectos éticos}

La investigación respetó los postulados establecidos por la Declaración de Helsinki (revisión de 2013) y los estándares éticos sugeridos para investigaciones en ciencias del deporte (Harris \& Atkinson, 2013). Esta investigación fue aprobada por la Comisión de Ética de la Universidad de Murcia (cuatro de diciembre de dos mil dieciocho), como parte de una tesis doctoral que se está desarrollando en el Departamento de Expresión Plástica, Musical y Dinámica de la Facultad de Educación.

\section{Instrumentos}

Programa de actividad física

Los participantes fueron sometidos a un programa de actividad física de ocho semanas (tabla 1), con dos sesiones semanales de 40-50 minutos de duración (40 minutos las semanas 1-2, 45 minutos las semanas 3-4, y 50 minutos las semanas 5-8), con 20 minutos de ejercicios de fuerza-resistencia muscular en circuito (tabla 2), con 1-2 minutos/ejerci- cio (1 minuto/ejercicio las semanas 1-4, 2 minutos/ejercicio las semanas 5-8) y 1 minuto/descanso, y 20 minutos de juegos basados en desafíos cooperativos (Fernández-Río \& Velázquez, 2005) (tabla 3). Las semanas 3-8 incluyeron un juego de recuperación basado en la resolución de un problema con bajo nivel de compromiso motor. El programa fue sometido a evaluación por tres jueces expertos (doctores en

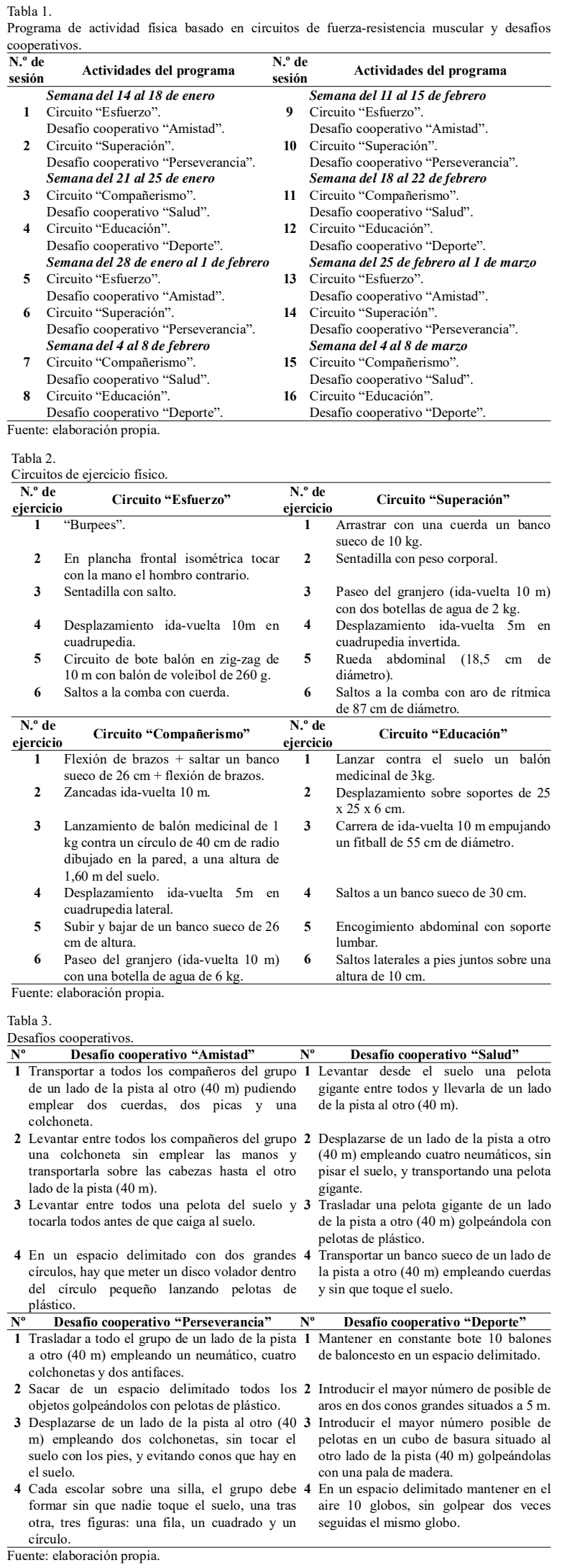

2 Levantar entre todos los compañeros del grupo 2 Desplazarse de un lado de la pista a otro una colchoneta sin emplear las manos y $(40 \mathrm{~m})$ empleando cuatro neumáticos, sin transportarla sobre las cabezas hasta el otro pisar el suelo, y transportando una pelota lado de la pista $(40 \mathrm{~m})$.

3 Levantar entre todos una pelota del suelo y 3 Trasladar una pelota gigante de un lado tocarla todos antes de que caiga al suelo. de la pista a otro $(40 \mathrm{~m})$ golpeándola con pelotas de plástico.

4 En un espacio delimitado con dos grandes 4 Transportar un banco sueco de un lado de círculos, hay que meter un disco volador dentro la pista a otro $(40 \mathrm{~m})$ empleando cuerdas del círculo pequeño lanzando pelotas de y sin que toque el suelo.

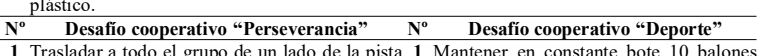
a otro $(40 \mathrm{~m})$ empleano de un ladóte cuatro de baloncesto en un espacio delimitado. colchonetas y dos antifaces.

2 Sacar de un espacio delimitado todos los 2 Introducir el mayor número de posible de objetos golpeándolos con pelotas de plástico. aros en dos conos grandes situados a $5 \mathrm{~m}$. 3 Desplazarse de un lado de la pista al otro (40 3 Introducir el mayor número posible de m) empleando dos colchonetas, sin tocar el pelotas en un cubo de basura situado al suelo con los pies, y evitando conos que hay en otro lado de la pista $(40 \mathrm{~m})$ golpeándolas el suelo.

4 Cada escolar sobre una silla, el grupo debe 4 En un espacio delimitado mantener en el formar sin que nadie toque el suelo, una tras aire 10 globos, sin golpear dos veces otra, tres figuras: una fila, un cuadrado y un seguidas el mismo globo. círculo.

Fuente: elaboración propia. 
ciencias de la actividad física y el deporte) con la finalidad de realizar los ajustes necesarios. Las sesiones se plantearon desde una perspectiva lúdica y en un clima de confianza (Baños \& Arrayales, 2020; Cabello, Moyano, \& Tabernero, 2018; Muñoz-Arroyave, Lavega-Burgués, Costes, Damian, $\&$ Serna, 2020). Cada semana los participantes fueron organizados en equipos heterogéneos de 4-5 escolares. Para favorecer la participación activa y la mayor intensidad posible, tanto los circuitos como los desafíos cooperativos fueron diseñados en forma de competición sin oposición, de manera que un mayor número de repeticiones o la resolución efectiva de los retos motrices indicó un mejor rendimiento. La intensidad de las sesiones se estimó con una adaptación de Sañudo \& del Hoyo (2007) del instrumento Children's OMNI scale of perceived exertion (Robertson et al., 2000). Se consideró como aspecto primordial la adherencia al programa motivando a todos los escolares para finalizarlo entregando cada semana insignias y diplomas por su esfuerzo y capacidad de superación (Zueck, Alonso, Margarita, \& Irigoyen, 2020).

\section{Atención}

La atención fue medida con el Test de Percepción de Semejanzas y Diferencias (Test de Caras) de Thurstone \& Yela (2012). Este instrumento consta de 60 elementos gráficos (imágenes-estímulo), cada uno de ellos formados por tres dibujos esquemáticos de caras con trazos simples, dos de las tres caras son iguales. La tarea consiste en tachar aquella que es diferente, y continuar con el resto de elementos. El tiempo máximo de la tarea es de tres minutos. Es un test de discriminación, semejanzas y diferencias en el que la velocidad de procesamiento y la atención selectiva desempeñan un papel importante. El Test de Caras ha sido empleado en investigaciones con escolares a partir de los seis años, y facilita la valoración de las habilidades perceptivoatencionales en escolares con y sin problemas de atención e hiperactividad (Monteoliva, Carrada, \& Ison, 2017; Monteoliva, Ison, \& Pattini, 2014; Rodríguez, Quinteros, Castro, \& Castro, 2008). En el diseño original del test se registró un coeficiente á $=.95$; en el estudio de validez se observó que la varianza se distribuía, sobre todo, entre los factores de velocidad de percepción e inteligencia espacial (Thurstone \& Yela, 1985). Este test ha sido analizado recientemente dando como resultado nuevos indicadores para la evaluación atencional (Monteoliva et al., 2017).

En este estudio se han propuesto las siguientes variables:

(1) Aciertos: figuras iguales al modelo -correctamente señaladas-.

(2) Errores de comisión (errores): figuras señaladas que difieren del modelo -incorrectamente señaladas-.

(3) Errores de omisión (omisiones): figuras similares al modelo no señaladas.

(4) Control inhibitorio: ratio de la diferencia entre aciertos y errores, dividido entre la suma de aciertos y errores. Esta variable ha sido descrita como una medida del control de la impulsividad, siendo más efectiva que la medición simple del número de figuras señaladas (Crespo-Eguílaz, Narbona, Peralta, \& Repáraz, 2006).

$$
\mathrm{CI}=\frac{\mathrm{A}-\mathrm{E}}{\mathrm{A}+\mathrm{E}}
$$

donde (CI) es el control inhibitorio, (A) es el número de aciertos y (E) los errores de comisión.

(5) Eficacia atencional: permite medir con exactitud la capacidad para discriminar estímulos dentro de un conjunto de estímulos semejantes (Ison \& Carrada, 2011).

$$
\mathrm{EA}=\frac{\mathrm{A}}{\mathrm{A}+\mathrm{E}+0}
$$

donde (EA) es la eficiencia atencional, (A) es el número de aciertos, (E) los errores de comisión y (O) los errores de omisión.

\section{Procedimiento}

El trabajo de campo fue desarrollado por un investigador principal, cuatro exploradores colaboradores previamente entrenados (graduados en ciencias del deporte) y el docente de educación física del centro. Por cuestiones prácticas y debido a la naturaleza del estudio (intervención centrada en la realidad y contexto escolar) los participantes fueron divididos en los grupos naturales de clase. El test de atención pretest y postest fue administrado a las $10^{`} 00$ horas, por el investigador principal, en un aula sin distracciones, con la suficiente privacidad y libertad en la cumplimentación. El grupo de tercero fue sometido a la intervención martes y jueves, y el grupo de cuarto miércoles y viernes. Todas las sesiones se desarrollaron desde las 10'40 hasta las 11'20 horas con el objetivo de interrumpir lo menos posible la dinámica del centro, y evitar el cansancio de las sesiones lectivas tras el recreo escolar.

\section{Análisis estadístico}

Se realizaron análisis descriptivos y de normalidad (Shapiro-wilk). Las diferencias entre medias pre-post se estudiaron con la prueba de Wilcoxon. Se empleó el programa SPSS (v.23.0, Chicago, Illinois, EE.UU.) fijando el nivel de significación en $p<.05$.

\section{Resultados}

Como análisis inicial se compararon las medias en las variables de atención en las medidas pre y post detectándose diferencias significativas en aciertos $(p<.001)$, errores $(p$ $=.012)$, omisiones $(p<.001)$, control inhibitorio $(p<.001) \mathrm{y}$ eficiencia atencional $(p<.001)($ tabla 4$)$.

Tabla 4 .

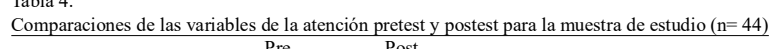

\begin{tabular}{lccccc}
\hline & Pre & Post & & & \\
& $\mathrm{M} \pm \mathrm{DE}$ & $\mathrm{M} \pm \mathrm{DE}$ & Dif· medias & $\mathrm{Z}$ & $p$ valor \\
\hline Aciertos & $26.9 \pm 6.5$ & $34.1 \pm 8.7$ & -7.2 & -4.89 & $<.001$ \\
Errores & $4.4 \pm 3.1$ & $3,3 \pm 4.6$ & 1.1 & -2.51 & .012 \\
Omisiones & $28.7 \pm 8.1$ & $20.9 \pm 9.8$ & 7.8 & -4.62 & $<.001$ \\
Control inhibitorio & $31.3 \pm 7.9$ & $37.4 \pm 9.6$ & -6.2 & -3.83 & $<.001$ \\
Eficacia atencional & $.44 \pm .11$ & $.59 \pm .14$ & -.15 & -5.17 & $<.001$ \\
\hline M $\pm \mathrm{DE}=$ media \pm desviación estándar. Fuente: elaboración propia. & &
\end{tabular}

Como análisis complementario se realizó una comparación de medias pre y post con el objetivo de verificar si estas diferencias se mantenían al dividir la muestra según sexo y edad (tablas 5 y 6). En la muestra de niños se encontraron diferencias en aciertos $(p<.001)$, errores $(p=.014)$, omisiones $(p=.001)$, control inhibitorio $(p=.034)$ y eficiencia atencional $(p=.001)$. En la muestra de niñas los resultados 
arrojados mostraron diferencias significativas en aciertos ( $p$ $<.001)$, omisiones $(p=.001)$, control inhibitorio $(p=.001)$ y eficiencia atencional $(p<.001)$. Al dividir la muestra según la edad las diferencias se mantuvieron. Así, en la muestra de 9 años se observaron diferencias significativas en aciertos $(p$ $=.007)$, omisiones $(p=.006)$, control inhibitorio $(p=0,049) \mathrm{y}$ eficiencia atencional $(p=.002)$. En la muestra de 10 años se encontraron diferencias significativas en aciertos $(p<.001)$, errores $(p=.006)$, omisiones $(p<.001)$, control inhibitorio $(p$ $=.001) \mathrm{y}$ eficiencia atencional $(p<.001)$.

\begin{tabular}{|c|c|c|c|c|c|}
\hline & $\begin{array}{l}\text { Pretest } \\
\mathrm{M} \pm \mathrm{DE}\end{array}$ & $\begin{array}{l}\text { Postest } \\
\mathrm{M} \pm \mathrm{DE}\end{array}$ & $\begin{array}{c}\text { Dif- } \\
\text { medias }\end{array}$ & $\mathrm{Z}$ & $p$ valor \\
\hline \multicolumn{6}{|l|}{ Varones } \\
\hline Aciertos & $27.2 \pm 6.9$ & $33.1 \pm 9.2$ & -5.9 & -3.39 & .001 \\
\hline Errores & $4.5 \pm 3.4$ & $3.3 \pm 5.5$ & 1.2 & -2.45 & .014 \\
\hline Omisiones & $28.5 \pm 8.9$ & $21.5 \pm 10.9$ & 7.0 & -3.21 & .001 \\
\hline Control inhibitorio & $31.5 \pm 8.7$ & $36.2 \pm 10.4$ & 4.7 & -2.06 & .039 \\
\hline Eficacia atencional & $.45 \pm .11$ & $.57 \pm .16$ & -.12 & -3.87 & $<.001$ \\
\hline \multicolumn{6}{|l|}{ Mujeres } \\
\hline Aciertos & $26.6 \pm 6.2$ & $35.4 \pm 8.0$ & 8.8 & -3.51 & $<.001$ \\
\hline Errores & $4.5 \pm 2.7$ & $3.4 \pm 3.3$ & 1.1 & -1.04 & .295 \\
\hline Omisiones & $29.0 \pm 7.3$ & $20.2 \pm 8.7$ & 8.8 & -3.42 & .001 \\
\hline Control inhibitorio & $30.8 \pm 7.3$ & $38.7 \pm 8.5$ & 7.9 & -3.27 & .001 \\
\hline Eficacia atencional & $.44 \pm .11$ & $.60 \pm .13$ & -.16 & -3.51 & $<.001$ \\
\hline \multicolumn{6}{|c|}{$\mathrm{M} \pm \mathrm{DE}=$ media \pm desviación estándar. Fuente: elaboración propia. } \\
\hline \multicolumn{6}{|l|}{ Tabla 6.} \\
\hline \multicolumn{6}{|c|}{$\begin{array}{l}\text { Valores de los parámetros de la atención pretest y postest para la muestra de escolares de } 9 \\
\text { años }(\mathrm{n}=23) \text { y } 10 \text { años }(\mathrm{n}=21) \text {. }\end{array}$} \\
\hline & Pretest & & & & \\
\hline & $\mathrm{M} \pm \mathrm{DE}$ & $\mathrm{M} \pm \mathrm{DE}$ & medias & Z & $p$ valor \\
\hline \multicolumn{6}{|l|}{9 años } \\
\hline Aciertos & $26.5 \pm 5.0$ & $30.7 \pm 6.3$ & -4.2 & -2.71 & .007 \\
\hline Errores & $4.6 \pm 2.9$ & $4.7 \pm 5.8$ & -.1 & -.83 & .406 \\
\hline Omisiones & $29.0 \pm 6.5$ & $22.1 \pm 9.9$ & 6.9 & -2.74 & .006 \\
\hline Control inhibitorio & $31.0 \pm 6.2$ & $35.3 \pm 8.6$ & -4.3 & -1.91 & .049 \\
\hline Eficacia atencional & $.44 \pm .08$ & $.54 \pm .12$ & -.10 & -3.13 & .002 \\
\hline \multicolumn{6}{|l|}{10 años } \\
\hline Aciertos & $27.3 \pm 8.0$ & $37.9 \pm 9.5$ & -10.6 & -3.96 & $<.001$ \\
\hline Errores & $4.2 \pm 3.2$ & $1.8 \pm 1.9$ & 2.4 & -2.77 & .006 \\
\hline Omisiones & $28.4 \pm 9.7$ & $19.6 \pm 9.8$ & 8.8 & -3.77 & $<.001$ \\
\hline Control inhibitorio & $31.4 \pm 9.7$ & $39.6 \pm 10.2$ & -8.2 & -3.35 & .001 \\
\hline Eficacia atencional & $.45 \pm .13$ & $.64 \pm .15$ & -.19 & -4.01 & $<0.001$ \\
\hline
\end{tabular}

\section{Discusión}

El objetivo del estudio fue analizar, en 24 niños y 20 niñas de 9-10 años de edad, si un programa de actividad física de alta intensidad con ejercicios de fuerza-resistencia muscular y juegos basados en desafíos cooperativos podía tener efectos sobre diversas variables que pueden describir las habilidades perceptivo-atencionales. Los principales hallazgos del estudio indican que el programa de actividad física tuvo un efecto positivo sobre la atención, especialmente, la atención selectiva, la eficacia atencional y el control inhibitorio, mostrándose igualmente consistentes cuando se realizó la comparación separada por sexo.

Estos resultados dan una respuesta precisa al objetivo de estudio y se sitúan próximos a aquellas investigaciones que sugieren una relación positiva entre actividad física y rendimiento cognitivo (Conde \& Tercedor, 2015), y entre actividad física y maduración mental en sus distintas dimensiones, siendo la atención selectiva una de ellas (Chaddock et al., 2010; Chaddock, Hillman, Pontifex, Johnson, Raine, \& Kramer, 2012); elementos todos ellos relacionados con una mejor salud mental y calidad de vida percibida (León, 2008; Rosa et al., 2018a,b,c).

Resultan asimismo de gran interés ya que contribuyen al desarrollo de conocimiento científico sobre el análisis de este fenómeno en la infancia; una etapa en la que el sistema nervioso central se muestra especialmente sensible a conductas que pueden influir sobre la neuroplasticidad cerebral y el funcionamiento cognitivo como, por ejemplo, los hábitos de actividad física (Fedewa \& Ahn, 2011; Maureira \& Flores, 2017).

Estos resultados podrían tener una relación con los mecanismos subyacentes de la activación de la función cognitiva inducida por el ejercicio físico regular y de alta intensidad (Chaddock et al., 2011), a través del incremento del flujo sanguíneo cerebral (Hillman, 2016), el aumento de la masa gris cerebral y la neurogénesis (Maureira, 2016), y el estado general de vigilancia tónica (Pérez et al., 2014).

A nivel metodológico, otras causas se podrían encontrar en las actividades empleadas en el programa; por un lado, ejercicios físico-lúdicos basados en CrossFit ${ }^{\text {(Gómez-Már- }}$ mol et al., 2014) que tienen un fuerte impacto sobre la condición física y donde los participantes informaron en un estudio de valores elevados de diversión, esfuerzo y aprendizaje (Sánchez-Alcaraz \& Gómez-Mármol, 2015); aunque otro estudio en la misma línea pero con sujetos jóvenes reportó de una disminución en la atención tanto selectiva como dividida (se incrementaron el número de errores y omisiones del instrumento del atención), posiblemente como consecuencia del aumento de lactato en sangre (Perciavalle et al., 2016). Por otro lado, se emplearon desafíos cooperativos, actividades planteadas como problemas motrices a resolver necesitando de una mayor implicación cognitiva a través de la reflexión, la toma de decisiones, cierto grado de creatividad y pensamiento divergente (Fernández-Río \& Méndez-Giménez, 2016).

Estos resultados se muestran en línea con algunos trabajos que han puesto de relieve la relación entre ejercicio físico y atención, ya sea en forma crónica (Alterburg et al., 2016; Reloba-Martínez et al., 2017) o en una sola sesión (Gallotta et al., 2015; Schmidt et al., 2015; Tine, 2014); y entre ejercicio físico, funcionamiento ejecutivo y velocidad de procesamiento (Gallego et al., 2015), concluyendo que el ejercicio físico puede ser una herramienta importante para estimular la atención y el funcionamiento cognitivo y, por ende, el rendimiento académico (Maureira \& Flores, 2017).

Resultados opuestos a los reportados en los estudios de Wilson et al. (2016) y Schmidt et al. (2016), tras implementar en el ámbito escolar intervenciones basadas en pausas activas de 10 minutos de duración, aunque este último observó que el compromiso cognitivo representaba la variable que mejor explicaba la mejora de la atención.

A pesar de que un estudio informó que no existían diferencias en tres tipos de intervención (ejercicio físico diario, meditación de atención plena y variaciones de la frecuencia cardiaca mediante biofeedback) en las mejoras sobre el control de la atención, el funcionamiento ejecutivo y la atención plena (De Bruin et al., 2016), los datos de otros trabajos incitan a pensar que las mejoras en la velocidad de procesamiento cognitivo, la capacidad atencional y la memoria de trabajo se producen tras la aplicación prolongada del programa de entrenamiento, lo que permite sugerir que la actividad física tiene efectos positivos sobre la atención cuando se observan cambios en el estado de condición física, sobre todo en el fitness cardiovascular (Gallego et al., 2015; Pontifex et al., 2011; Wu et al., 2011), reflejándose objetivamente en parámetros fisiológicos como el consumo máximo de oxígeno (Reloba-Martínez et al., 2017). 
Los resultados obtenidos hay que interpretarlos con cierta precaución ya que este estudio presenta diversas limitaciones. Entre otras, el diseño de muestreo, la escasa variabilidad en la composición de la muestra, la recopilación de datos mediante cuestionarios, la ausencia de variables de control como por ejemplo la condición física, y la valoración subjetiva de la intensidad de las sesiones. Estas limitaciones dificultan la capacidad de extrapolar los resultados a otras poblaciones de estudio, aunque también presenta algunas aportaciones. El diseño metodológico bien definido facilita la replicación del estudio con el objetivo de consolidar la evidencia científica disponible. El desarrollo de la intervención en la propia realidad y contexto escolar, con profesionales cercanos al ámbito educativo permite que docentes de enseñanza primaria puedan aplicar el programa con la finalidad de desarrollar las habilidades perceptivo-atencionales de su alumnado.

Con las precauciones señaladas se puede concluir que la realización de un programa de actividad física de 16 sesiones, de 40-50 minutos de duración/sesión, basado en ejercicios de fuerza-resistencia muscular y desafíos cooperativos podría tener efectos positivos sobre la atención selectiva y la eficacia atencional tanto en varones como en mujeres de 910 años de edad. La adherencia a un programa de ejercicios físico-lúdicos, con actividades en las que los participantes se puedan mostrar implicados en una tarea común motivadora, sin menoscabo de una elevada intensidad autopercibida podrían ser factores a tener en cuenta para el éxito de una intervención orientada a este objetivo, además de poder reportar resultados en otras facetas como la salud mental, el rendimiento académico y cognitivo.

\section{Referencias}

Altenburg, T., Chinapaw, M. \& Singh, A. (2016). Effects of one versus two bouts of moderate intensity physical activity on selective attention during a school morning in Dutch primary schoolchildren: A randomized controlled trial. Journal of Science and Medicine in Sport, 19(10), 820-824.

Ato, M., López, J. J. \& Benavente, A. (2013). Un sistema de clasificación de los diseños de investigación en psicología. Anales de Psicología, 29, 1038-1059. Doi: http://dx.doi.org/ 10.6018/analesps.29.3.178511.

Baños, R. \& Arrayales, E. (2020). Predicción del aburrimiento en la educación física a partir del clima motivacional. Retos, 38, 83-88.

Barnett, L. M., Van Beurden, E., Morgan, P. J., Brooks, L.O., \& Beard, J. R. (2010). Gender differences in motor skill proficiency from childhood to adolescence: A longitudinal study. Research Quarterly for Exercise and Sport, 81,162170.

Cabello, A., Moyano, M., \& Tabernero, C. (2018). Procesos psicosociales en Educación Física: actitudes, estrategias y clima motivacional percibido. Retos, 34, 19-24.

Chaddock, L., Hillman, C. H., Pontifex, M. B., Johnson, C. R., Raine, L. B., \& Kramer,A. F.(2012). Childhood aerobic fitness predicts cognitive performance one year later. Journal of sports science, 30(5), 421-430.

Chaddock,L.,Pontifex,M.B,Hillman,Ch.H., \& Kramer,A.F.(2011). A Review of the Relation of Aerobic Fitness and Physical Activity to Brain Structure and Function in Children. Journal of the International Neuropsychological Society, 17, 1-11. Chen, W., Zhang, Z., Callaghan, B., LaChappa, L., Chen, M., \& He,Z. (2017).Acute Effects of Aerobic Physical Activities on Attention and Concentration in School-aged Children. Biomed Journal of Scientific \& Technical Research, 1(5), 18.DOI: 10.26717/BJSTR.2017.01.000456.

Conde, M.A., \& Tercedor, P.(2015). La actividad física, la educación física y la condición física pueden estar relacionadas con el rendimiento académico y cognitivo en jóvenes. Revisión sistemática. Archivos de medicina del deporte: Revista de la Federación Española de Medicina del Deporte y de la Confederación Iberoamericana de Medicina del Deporte, 166, 100-109.

Crespo-Eguílaz, N., Narbona, J., Peralta, F. \& Repáraz, R. (2006). Medida de atención sostenida y del control de la impulsividad en niños: nueva modalidad de aplicación del Test de Percepción de Diferencias «Caras». Infancia y Aprendizaje, 29(2), 219-232.

de Bruin, E.I., van der Zwan, J.E., \& Bögels, S.M. (2016). ARCT Comparing Daily Mindfulness Meditations, Biofeedback Exercises, and Daily Physical Exercise on Attention Control, Executive Functioning, Mindful Awareness, SelfCompassion, and Worrying in Stressed Young Adults. Mindfulness(NY), 7(5), 1182-1192. doi: 10.1007/s12671-0160561-5.

Fedewa, A. L. \& Ahn, S. (2011). The effects of physical activity and physical fitness on children's achievement and cognitive outcomes: a meta-analysis. Research Quarterly for Exercise and Sport, 82, 521-535.

Fernández-Río, J., \& Méndez-Giménez,A.(2016). ElAprendizaje Cooperativo: Modelo Pedagógico para Educación Física. Retos, 29, 201-206.

Fernández-Rio, J. \& Velázquez, C. (2005). Desafíos físicos cooperativos. Retos sin competición para las clases de Educación Física. Sevilla: Wanceulen.

Folgado dos Santos, J.M., Petrica, J., Serrano, J., Batista, M., Honório, S., Lercas, A., \& Maia,L. (2020).Attention in physical education classes: Differences between colletive sports. Retos, 37, 129-133.

Goodway, J.D., Ozmun, J.C., \& Gallahue, D.L. (2020). Understanding motor development: infants, children, adolescents and adults (8th ed.). New York: McGraw Hill.

Gallego, V., Reigal, R., Hernández,A., \& Juárez, R. (2015). Efectos de la actividad física sobre el funcionamiento cognitivo en preadolescentes. Apunts. Educación Física y Deportes, 121(3), 20-27. http://dx.doi.org/10.5672/apunts.20140983.es.(2015/3).121.03

Gallotta, M., Emerenziani, G., Franciosi, E., Meucci, M., Guidetti, L. \& Baldari, C. (2015). Acute physical activity and delayed attention in primary school students. Scandinavian Journal Medicine Science Sports, 25(3), e331-338.

Giuliano, R. J., Karns, C.M., Neville, H. J. \& Hillyard, S. A. (2014). Early auditory evoked potential is modulated by selective attention and related to individual differences in visual working memory capacity. Journal of Cognitive Neuroscience, 26(12), 2682-2690.

Gómez-Mármol,A., Sánchez-Alcaraz,B.J., Pérez, M., \& Ribes,A. (2014). El CrossFit en la Educación Física Escolar. EmásF, Revista Digital de Educación Física, 30(5), 19-34.

Hillman, C. H. (2016). Aerobic fitness is associated with greater 
hippocampal cerebral blood flow in children. Developmental Cognitive Neuroscience, 20, 52-58.

Ison, M.S., Greco, C., Korzeniowski, C.\& Morelato, G.S. (2015). Selective attention: A comparative study on Agentine students from different socioeconomic contexts. Electronic Journal of Research in Educational Psychology, 13(2), 343 368. http://dx.doi.org/10.14204/ejrep.36.14092

Khan, N. \& Hillman, C. H. (2014). The Relation of Childhood Physical Activity andAerobic Fitness to Brain Function and Cognition: A Review. Pediatric Exercise Science, 26, 138146. Doi: http://dx.doi.org/10.1123/pes.2013-0125.

Korzeniowski, C. (2011). Evolutionary development of executive functioning and its relationship with school learning. Revista de Psicología, 7(13), 7-26.

León, B. (2008). Atención plena y rendimiento académico en estudiantes de enseñanza secundaria. European Journal of Education and Psychology, 1(3), 17-26.

López, G.F., López, L., \& Díaz,A. (2016). Efectos de un programa de actividad física en la calidad del sueño de escolares con TDAH. Revista Euroamericana de Ciencias del Deporte, 1(5), 19-26.

Maureira, F. \& Flores, E. (2016). Principios de neurobiopsicología para estudiantes de educación. Valencia: Obrapropia.

Monteoliva, J.M., Carrada, M., \& Ison, M. (2017). Test de percepción de diferencias: estudio normativo del desempeño atencional en escolares argentinos. Interdisciplinaria, 34(1), $39-56$.

Monteoliva, J.M., Ison, M.S., \& Pattini,A.E. (2014). Evaluación del desempeño atencional en niños: Eficacia, eficiencia y rendimiento. Interdisciplinaria, 31(2), 213-225.

Muñoz-Arroyave, V., Lavega-Burgués, P., Costes, A., Damián, S., \& Serna, J. (2020). Los juegos motores como recurso pedagógico para favorecer la afectividad desde la educación física. Retos, 38, 166-172.

Ortiz, R. \& Ramírez, M.L. (2020). Actividad física, cognición y rendimiento escolar: una breve revisión desde las neurociencias. Retos, 38.

Perciavalle, V., Marchetta, N., Giustiniani, S., Borbone, C., Perciavalle, V., Petralia, M.,... \& Coco, M. (2016). Attentive processes, blood lactate and CrossFit ${ }^{\circledR}$. Physician and Sportsmed, 44(4), 403-406.

Pérez, D. \& Guzmán, J.F. (2019). Predictores cognitivos de la intención de práctica y la percepción de las relaciones en el deporte: análisis de la pasión como mediadora. Retos, 36, 193-202.

Petersen, S.E. \& Posner, M.I. (2012). The attention system of the human brain: 20 years after. Annual Review Neuroscience, 35, 73-89. http://dx.doi.org/10.1146/annurev-neuro 062111150525.

Piaget, J. \& Inhelder, B. (2015). Psicología del niño. Madrid: Morata.

Pontifex, M. B., Raine, L. B., Johnson, C. R., Chaddock, L., Voss, M. W., Cohen, N. J.,... \& Hillman, C. H. (2011). Cardiorespiratory fitness and the flexible modulation of cognitive control in preadolescent children. Journal of Cognitive Neuroscience, 23, 1332-1345. Doi: http:// dx.doi.org/10.1162/jocn.2010.21528

Reloba-Martínez, S., Reigal-Garrido, R.E., Hernández-Mendo,A., Martínez-López, E. J., Martín-Tamayo, I., \& Chirosa-Ríos, L.J. (2017). Efectos del ejercicio físico extracurricular vigoroso sobre la atención de escolares. Revista de psicología del deporte, 26(2), 29-36.

Robertson, R.J., Goos, F.L., Boer, N.F., Peoples, J.A., Foreman, A.J., Dabayebeh, I.M.,. . . \& Thompkins, T. (2000). Children's OMNI scale of perceived exertion: mixed gender/race validation. Medicine \& Science in Sports \& Exercise, 32, 457-58.

Rodríguez, M. C., Alexey, E., Castro, R., \& Castro, F. M.(2008). Diseño y pilotaje de un programa de ejercicios físico-lúdicos para estimular la atención en niños de 8 a 10 años. Revista Iberoamericana de Educación, 47(1), 1-10.

Rosa,A., García-Cantó, E., \& Carrillo, P.J.(2018a). Weight status, physical activity and self-concept in primary school children. Journal of Human Sport and Exercise, 14(3), 515-526. doi:https://doi.org/10.14198/jhse.2019.143.03.

Rosa,A., García-Cantó, E., \& Carrillo, P. J.(2018b). Percepción de salud, actividad física y condición física en escolares. Cuadernos de Psicología del Deporte, 18(3), 179-189.

Rosa,A., García-Cantó, E., \& Pérez-Soto, J.J.(2018c). Condición física y bienestar emocional en escolares de 7 a 12 años. Acta colombiana de Psicología, 21(2), 282-291. doi: http:// www.dx.doi.org/10.14718/ACP.2018.21.2.13.

Rosa,A., García, E., \& Carrillo, P.J. (2019). Capacidad aeróbica y rendimiento académico en escolares de educación primaria. Retos, 35, 351-354.

Sánchez-Alcaraz, B.J. \& Gómez-Mármol,A. (2015). Percepción de esfuerzo, diversión y aprendizaje en alumnos de Educación secundaria, Sport TK: Revista Euroamericana de ciencias del deporte, 4(1), 63-68.

Sañudo, B. \& del Hoyo, M. (2007). La percepción de esfuerzo en edad escolar. Cultura Ciencia y Deporte, 3, 13-7.

Schmidt, M., Egger,F. \& Conzelmann,A. (2015). Delayed positive effects of an acute bout of coordinative exercise on children's attention. Perceptual and Motor Skills, 121(2), 431-446.

Slavin, R. (1992). Aprendizaje cooperativo. En C. Rogers y P. Kutnick (comps.). Psicología social de la escuela primaria. 247270. Barcelona: Paidós.

Thurstone, L. L. \& Yela, M.(1985). Test de percepción de diferencias (Caras). Madrid: TEA Ediciones.

Thurstone, L. L. \& Yela, M. (2012). Caras-R: test de percepción de diferencias-revisado: manual. Madrid: Tea.

Tine, M. (2014). Acute aerobic exercise: an intervention for the selective visual attention and reading comprehension of lowincome adolescents. Frontiers in Psychology, 5, 575.

Villa deGregorio, M., Ruiz,L.M., \& Barriopedro, M.I.(2019).Análisis de las relaciones entre el Trastorno en el Desarrollo de la Coordinación(TDC/DCD) y el Trastorno por Déficit de Atención e Hiperactividad (TDAH) en la edad escolar. Retos, 36, 625-632.

Wilson, A., Olds, T., Lushington, K., Petkov, J. \& Dollman, J. (2016). The impact of 10-minute activity breaks outside the classroom on male students' on-task behaviour and sustained attention: a randomised crossover design. Acta Paediatrica, 105(4), e181-188.

Wu, C., Pontifex, M., Raine, L., Chaddock, L., Voss, M., Kramer,A. \& Hillman, C.(2011). Aerobic fitness and response variability in preadolescent children performing a cognitive control task. Neuropsychology, 25(3), 331-341

Zueck, M., Alonso, A., Margarita, J., \& Irigoyen, H.E. (2020). Satisfacción en las clases de Educación Física y la intencionalidad de ser activo en niños del nivel de primaria. Retos, 37,33-40. 